\title{
Major of engineering in normal university of one hundred years
}

\author{
Ping Wang ${ }^{1}$ \\ College of Photonic and Electronic Engineering \\ FuJian Normal University \\ Fuzhou,China \\ pwang@fjnu.edu.cn \\ Guodong $\mathrm{Su}^{3}$ \\ College of Photonic and Electronic Engineering \\ FuJian Normal University \\ Fuzhou,China \\ 693826334@qq.com
}

\author{
Bingjie Chen ${ }^{2}$ \\ College of Photonic and Electronic Engineering \\ FuJian Normal University \\ Fuzhou,China \\ 1099710771@qq.com \\ Bin Chen ${ }^{4}$, \\ College of Photonic and Electronic Engineering \\ FuJian Normal University \\ Fuzhou,China \\ chenbinlove2009@163.com
}

\begin{abstract}
This paper narrated the origins of the environment development and things about engineering courses in our university. It analyzed issues about how to improve personal training and correct faultiness in current situation. According to the engineering specific area, using “ TCP/IP protocol principle and network programming” as example, this paper discussed the methods about accelerating the process of electronic information engineering and stated the reason why it is necessary and significant to improve our teaching methods. Finally, it concluded the process and achievements in this decade.
\end{abstract}

Keywords-component; Normal Universities;Characteristic Engineering;Electronics information

\section{I.THE SUBJECT DEVELOPMENT}

National prosperity depends on talents, talents prosperity depends on education, education prosperity depends on the teacher, the teacher prosperity depends on normal education [1]. "Normal university" only literally should understand to foster "just one teacher and model of the" talent school, so the domestic various normal university usually has a long history, the teaching standard, in the society has high credibility.

Fujian normal university after one hundred years of development, now has 71 undergraduate, became literature, history and philosophy, science, engineering,teaching, and the method, pipe, agriculture, art and multidisciplinary coordinated development of

the education pattern, already beyond the "normal" category in fact become a comprehensive university.

Physics is the study of material structure and the interaction and movement basic rule primary discipline, subordinate theoretical physics, particle physics and nuclear physics, atomic and molecular physics, condensed matter physics, plasma physics, acoustic, optical and radio physics and so on several secondary discipline. In the physical development of the discipline, from the beginning is mainly for physical education major students, by 1998 expansion additional application of electronic technology education professional training teaching teachers (science). Because of the province after teachers increasingly saturated, the electronic technology education professional training to 2005 in 2000 and changed the check for electronic information engineering students (engineering), and then open and network engineering, optical engineering, optical engineering, communication engineering major in engineering.

\section{II.CHARACTERISTICS CHANGE}

Physical and engineering belong to different subject categories, heavy science theory and method, and heavy engineering technology and process. Engineering is the application of the principle of basic science, combined with the production practice of accumulated experience and technology development of subject, the main cultivation of practical application ability of technical personnel.

The science teacher position will be responsible for the teaching and the task of knowledge, a process of training students be theoretical basis

Development of instrument, write, expression, communication are requirements [2]. The engineer position engineering will be responsible for project implementation and technology research and development tasks, training process must pay attention to practical ability.

Only from Fujian normal university of electronic information engineering specialty name, the society will because the school's one hundred years of history and trust it's quality of teaching, but unit of choose and employ persons also because "normal" two characters and worry about engineering was born the ability to solve practical problems. In the heart also really do exist the adverse factor:

(1). Heavy theory light practice: long-term science normal class training experience formation of habitual thinking in a certain degree of influence on engineering development, such as: the theory of training program to the needs of society and contact close enough, do not advocate teacher outside part-time or office tradition also restrict 
teacher tracking new technology and the improvement of ability, hope all practice base practice can also affect students' employment rate.

(2). The lack of leader: high quality engineering talents in the enterprise have very favorable treatment, but the lack of capital school talent introduction mechanism can't provide the corresponding treatment, this big orders of magnitude difference even make us ashamed to open. Take a look at the level to apply for the Ph.D., because professional characteristics, in some professional people, electronic information to apply for $\mathrm{PhD}$ original little, often also in the interview to talk a few times to give up after each other.

(3). Heavy research light teaching: the current environment evaluation system of teaching component less poor, of a teacher's level of scientific research ability, and is mainly engineering application type paper level is generally not high, which directly affect the engineering professional teachers' professional title evaluation. Plus, because of hot professional student/teacher ratio is large, heavy teaching task that the teachers did not have much more energy for scientific research and teaching reform, the superior check the opinions of the back almost all focus on professor proportion and scientific research, teaching reform project is not the two aspects.

\section{III.ENGINEER TRAINING}

In science the atmosphere grow up engineering major, how to highlight the engineering characteristics, develop distinctive and improve competitiveness? Should is our professional teachers diligent in thinking problem and long-term effort direction.

In the early construction of electronic information engineering specialty, we will focus on the laboratory construction, to the professional laboratory building area of 3134 square meters, instruments and equipment of RMB 7.8 million yuan. At the same time, constantly adjusting is different from the normal kind of curriculum structure [3], increase the proportion of practice class, practice credits is greater than $30 \%$ of the total credit, in order to improve the employment rate in the professional direction increase various module for students to take as an elective course. The whole training process using the series means to strengthen the student beginning ability:

(1). Classes teacher: from freshman year began to shift to appoint a professional teachers and graduate students for vice class teacher, mainly to help solve the problem of learning, science and technology.

(2). Students 10th science day: 10th science day once a year to decorate project tasks, display comparison student's science and technology works, the commendation advanced individual, the purpose of this paper is to keep the fine tradition of institutionalization.

(3). Innovation laboratory: to provide students with good science and technology activity places, with independent project of scientific research and to participate in all kinds of high level professional competition as the means, cultivate engineering quality and innovation spirit.

(4). To promote teachers' scientific research: encourage teachers' active declaration research (vertical and horizontal), only the teacher first, updating, and master the new technology can teach good students, and at the same time, attracting outstanding students to participate in scientific research project, lead students science and technology activities.

(5). Pay attention to the graduation design: design work is divided into hardware design class, software design class, engineering design class three, teachers by the school and enterprise of two parts.

\section{IV.PROFESSIONAL CHARACTERISTICS}

Electronic and information engineering is a broad caliber engineering major, coherent main subject for: electronic science and technology, information and communication engineering, computer science and technology. The professional obtain employment is in good condition, post involved in telecommunications, energy, transportation, finance, economics, trade, military, science, education, social security, all levels of government agencies, and other fields. In the surrounding university are set up the professional situation, how to improve the competitiveness? Professional characteristics is very important!

Feature is a thing or a significant things different from other things style, form, which is the things of the emergence and development of certain specific environmental factors, it is their unique things. The photoelectric and information engineering college by some related discipline system, research centers, with the provincial, the ministry of education key laboratory, the related interdisciplinary rich experience in teaching faculty and outstanding scientific research platform, is the professional development has laid a good foundation.

We keep the direction of the popularization of the basis of running with their history and resource advantages put forward: keep "computer technology of electronic and information engineering application and research and development", "management information technology based software engineering application and research and development", and focuses on the traditional "application of the embedded system and research and development" as the main direction of the characteristics. Popular explanation is based on embedded system is partial hardware training direction, said of the embedded system platform contains single-chip microcomputer technology, ARM technology, FPGA technology, DSP technology, virtual instrument technology five hardware platform.

\section{V.TEACHING REFORM}

Einstein once said: "interest is the best teacher." Electronic information or related professional knowledge update quickly, students if there is no this professional strong study interest, it is hard to imagine the students after graduation can meet the demand of talents social needed [4]. In the teaching process is the most important still is to improve the students' interest in professional knowledge.

In today's rapid development of the information age, the electronic information technology at home and abroad and 
the development of science and technology plays a very important role in the [5], the electronic information courses also has its own particular characteristics and nature of [6]. Internet originated in the United States, is the biggest influence in the world, and the people most closely related, changed People's Daily communication and the communication mode of communication network, the network is the TCP/IP protocol. Now, all kinds of computer network, protocol type of courses many, although they different emphasis but also with many, including the TCP/IP protocol principle and network programming "for the core principle about agreement more important, in our school, this course is electronic and information engineering, network engineering major required courses and many related professional elective courses.

The problem is the translation version of the Chinese teaching materials are mostly exist statement obscure, wrong a lot of problems. And the core content of this course is composed of 0,1 see touched agreement, teaching in some key points and difficulties, with the traditional teaching method interpretation and time-consuming, the effect is not good, should study supporting the corresponding teaching means, and the concrete measures are as follows:

(1). According to relevant course content repetition, revised curriculum system, to make the curriculum standard, the standard experimental program process, expand the course, each class hour will be cut down the network technology ", "the OSI protocol", "integrated wiring technology" part of the course content into the course teaching, rise to digest the good effect.

(2). According to the teaching material is not ideal, it is only through the teachers more than their reading, diligently practice. Teaching theory will be part of the focus on the OSI protocol and TCP/IP layer, application layer in the theory of interpretation teaching simple will focus on practical programming link. Generally speaking, a good course, at least after three rounds of the teaching experience, a clear understanding to the teaching order and emphasis.

(3). In theory how to contact the practice problems, due to the course is very suitable for the project drive method to improve the students' understanding and interest, the whole teaching process are in various application for project, three people a group, each network programming point of view is different, but in the end can be consolidated into a perfect application.

(4). According to the agreement is difficult to understand, as shown in figure 1 shows, explain in routing principle stage, the network simulation software to understand routing principle; As shown in figure 2 shows, the interpretation agreement stage, using caught analysis software confirmed agreement; So that the original relative tasteless 0,1 agreement theory become lively and interesting.

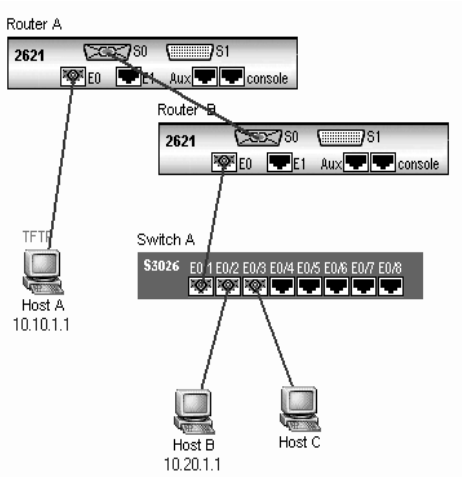

Figure1 network simulation software

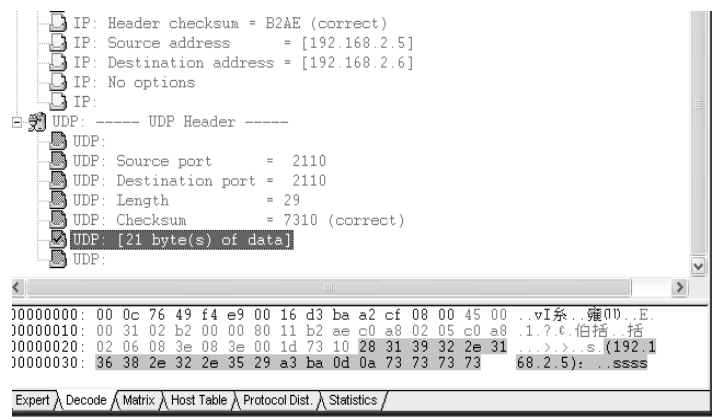

Figure 2 caught analysis software

Practice has proved that through the reform of teaching methods and means, students on the course have a strong interest in, the master in digital communication system related agreement knowledge and theory, familiar with the network programming methods and means, strong analysis and the ability to solve practical problems, cultivate a good professional quality and innovation consciousness.

\section{VI.CONSTRUCTION EFFECT}

In the joint efforts of all teachers, electronic information engineering construction has obtained certain result: in 2005, upgraded to the school subjects (signal and information processing), 2005 approved communication and information systems, computer application technology, radio physics, nuclear physics four master degree program, in 2007 was assessed at provincial electrical and electronic experimental teaching demonstration center, 2008 was assessed at provincial professional characteristics.

In more than twenty full-time teachers: forty years old of the following young teachers accounted for $48 \%$, with the professional formal education teachers accounted for $85 \%$, with the master degree or above accounted for $48 \%$ of teachers, with doctor's degree of teachers accounted for $22 \%$, high title teachers accounted for $35 \%$. In recent years the high level academic papers published hundreds of articles, such as by EI and ISTP included nearly international papers, patented nearly items, the writing materials, dozens of the host to participate in research projects for more than items, the provincial science and technology progress prize many. 
Ten years, electronic information engineering professional trained graduate students 899 people, one's deceased father grind rate at about $20 \%$, an employment rate $>90 \%$, the professional students of nearly 80 people win the national (province) "challenge cup", the national electronic design contest (province), such as the first, second and third.

\section{VII.CONCLUSION}

Specialty construction is a long-term process, in the present size environment may come across a lot of difficulties, need all the teachers' hard work and selfless dedication, only follow the law of engineering education, closely related social needs, concise unique professional direction, to highlight the characteristics of a school, realizing training subject basis down-to-earth, practice ability of high quality engineering senior talents training target [7].

\section{ACKNOWLEDGEMENT}

Fund Project: Fujian Electronic Information Engineering Specialty Construction Point (L200802008); "TCP / IP Principles and network programming teaching methods and reform of teaching methods (I201007020)

\section{REFERENCES}

[1] Qu Tiehua, FanTao. Higher normal education system in the late qing dynasty free characteristics analysis [J]. Journal of hebei normal university, 2009, (3) : 23-27.

[2] ZhangYanFen. Analyses the development of normal colleges recessive course [J].journal of education theory and practice, 2010, 21 (3) : -

[3] Soloing. In one hundred normal course cultural tradition and the characteristic [J].journal of Jiangsu education research, 2010, (4) : - 23.

[4] XiongJieFeng, electronic class undergraduate course teaching in teaching students according to their aptitude thinking [J]. China science and technology information, 2008, (12) : 252-253.

[5] QinLi, ZhangWenDong, XiongJiJun, Liu Jun, Ren Yongfeng, Liu Wenyi. Electronic professional students' innovative and practical ability training mode analysis [J]. Journal of bei university, 2008, (6) : - 29.

[6] Jiang Shuhua, Li Mingqiu, Zhang Chen clean. Electronics course examination system reform of the exploration and practice of science and technology information [J]. J, 2009, : 252-253.

[7] Wu Ling tin, and Zhan Jie, Zhou Renlong. Electronic information science and technology major curriculum system characteristics [J].journal of contemporary education theory and practice, 2010, 2 (1) : 105-107. 